\title{
New bounds for the sum of powers of normalized Laplacian eigenvalues of graphs*
}

\author{
Gian Paolo Clemente, Alessandra Cornaro \\ Catholic University, Department of Mathematics, Finance and Econometrics, Milan, Italy
}

Received 5 May 2015, accepted 26 May 2016, published online 25 August 2016

\begin{abstract}
For a simple and connected graph, a new graph invariant $s_{\alpha}^{*}(G)$, defined as the sum of $\alpha$ powers of the eigenvalues of the normalized Laplacian matrix, has been introduced by Bozkurt and Bozkurt (2012). Lower and upper bounds for this index have been proposed by the authors. In this paper, we localize the eigenvalues of the normalized Laplacian matrix by adapting a theoretical method, proposed in Bianchi and Torriero (2000), based on majorization techniques. Through this approach we derive upper and lower bounds of $s_{\alpha}^{*}(G)$. Some numerical examples show how sharper results can be obtained with respect to those existing in literature.
\end{abstract}

Keywords: Graphs, majorization, topological indices, bounds.

Math. Subj. Class.: 05C35, 05C05, 05C50

\section{Introduction}

Among the various indices in Mathematical Chemistry, a whole new family of descriptors $s_{\alpha}^{*}(G)$, defined as the sum of $\alpha$ powers of the eigenvalues of the normalized Laplacian matrix, has been proposed by Bozkurt and Bozkurt in [7]. These authors found a number of bounds for arbitrary $\alpha$ and particularly for $\alpha=-1$, which is the case of the degree Kirchhoff Index. Recently, Bianchi et al. proposed a variety of lower and upper bounds for $s_{\alpha}^{*}(G)$ in [1] and for the Kirchhoff Index in [2] derived via majorization techniques. In particular, the authors showed that it is possible to obtain tighter results taking into account additional information on the localization of the eigenvalues of proper matrices associated to the graph. From a theoretical point of view, some well-known inequalities on the localization of real eigenvalues have been provided in literature and they can be used to compute

\footnotetext{
*We thank Monica Bianchi, Anna Torriero and an anonymous referee for their valuable comments and suggestions.

E-mail addresses: gianpaolo.clemente@unicatt.it (Gian Paolo Clemente), alessandra.cornaro@unicatt.it (Alessandra Cornaro)
} 
the above mentioned bounds.

Alternative inequalities involving the localization of some eigenvalues of the transition matrix of the graph have been numerically computed in [9] and [10] by applying a theoretical methodology proposed in Bianchi and Torriero [5] based on nonlinear global optimization problems solved through majorization techniques. By means of these results, tighter lower bounds for the Kirchhoff Index for some classes of graphs have been derived in [9].

The original contribution of this paper is to exploit this fruitful theoretical method (see [5]) with the aim to provide some formulae that allow us to compute lower bounds for the first and the second eigenvalues of the normalized Laplacian matrix in a fairly straightforward way. These limitations on the eigenvalues are then used to assess bounds for $s_{\alpha}^{*}(G)$ proposed in [1]. We then obtain new bounds for $s_{\alpha}^{*}(G)$ considering both non-bipartite and bipartite graphs.

In Section 2 some preliminaries on graph theory are given. Furthermore, the definition of $s_{\alpha}^{*}(G)$ and the existing bounds on this index are presented. In Section 3 we describe the nonlinear optimization problem based on majorization techniques. This methodology, useful for our analysis, allows us to localize the first and second eigenvalues of the normalized Laplacian matrix. Lower bounds of these normalized Laplacian eigenvalues have been obtained in Section 4. We prove that our limitation on the first normalized Laplacian eigenvalue is always sharper than the existing one for non-complete graph. By means of this result, we provide bounds on $s_{\alpha}^{*}(G)$ tighter than those given in [7]. Finally, in Section 5 a numerical comparison for bipartite and non-bipartite graphs is reported.

\section{Notations and preliminaries}

In this section we first recall some basic notions on graph theory. For more details refer to [17].

Let $G=(V, E)$ be a simple, connected, undirected graph where $V=\{1,2, \ldots, n\}$ is the set of vertices and $E \subseteq V \times V$ the set of edges, $|E|=m$.

The degree sequence of $G$ is denoted by $\pi=\left(d_{1}, d_{2}, . ., d_{n}\right)$ and it is arranged in nonincreasing order $d_{1} \geq d_{2} \geq \cdots \geq d_{n}$, where $d_{i}$ is the degree of vertex $i$. It is well known that $\sum_{i=1}^{n} d_{i}=2 m$ and that if $G$ is a tree, i.e. a connected graph without cycles, $m=n-1$. Let $A(G)$ be the adjacency matrix of $G$ and $D(G)$ be the diagonal matrix of vertex degrees. The matrix $L(G)=D(G)-A(G)$ is called Laplacian matrix of $G$, while $\mathcal{L}(G)=D(G)^{-1 / 2} L(G) D(G)^{-1 / 2}$ is known as normalized Laplacian matrix. Let $\mu_{1} \geq \mu_{2} \geq \ldots \geq \mu_{n}$ be the (real) eigenvalues of $L(G)$ and $\lambda_{1} \geq \lambda_{2} \geq \ldots \geq \lambda_{n}$ be the (real) eigenvalues of $\mathcal{L}(G)$. The following properties of spectra of $L(G)$ and $\mathcal{L}(G)$ hold:

$$
\begin{gathered}
\sum_{i=1}^{n} \mu_{i}=\operatorname{tr}(L(G))=2 m ; \quad \mu_{1} \geq 1+d_{1} \geq \frac{2 m}{n} ; \mu_{n}=0, \mu_{n-1}>0 . \\
\sum_{i=1}^{n} \lambda_{i}=\operatorname{tr}(\mathcal{L}(G))=n ; \sum_{i=1}^{n} \lambda_{i}^{2}=\operatorname{tr}\left(\mathcal{L}^{2}(G)\right)=n+2 \sum_{(i, j) \in E} \frac{1}{d_{i} d_{j}} ; \lambda_{n}=0 ; \lambda_{1} \leq 2 .
\end{gathered}
$$

Our aim is the analysis of a particular topological index, $s_{\alpha}^{*}(G)$. In particular, Zhou (see [18]) proposed the index:

$$
s_{\alpha}(G)=\sum_{i=1}^{n-1} \mu_{i}^{\alpha}, \alpha \neq 0,1,
$$


defined as the sum of the $\alpha$-th power of the non-zero Laplacian eigenvalues of a graph $G$.

Over the last years this index and its bounds have been intensely studied: Zhou (see [18]) established some properties of $s_{\alpha}(G)$ and some improvements have been provided in [14], [16], [19] and [20]. In [3], taking into account the Schur-convexity or Schurconcavity of the functions $s_{\alpha}(G)$ for $\alpha>1$ and $\alpha<0$ or $0<\alpha<1$ respectively, the same bounds as in [18] have been derived. Furthermore, considering additional information on the localization of the eigenvalues, the authors provide also sharper bounds.

Bozkurt and Bozkurt in [7] introduced parallely to [18] the following new graph invariant:

$$
s_{\alpha}^{*}(G)=\sum_{i=1}^{n-1} \lambda_{i}^{\alpha}, \alpha \neq 0,1,
$$

characterized as the sum of the $\alpha$-th power of the non-zero normalized Laplacian eigenvalues of a graph. Several properties of this index have been proposed in [7] and some lower and upper bounds for a connected graph have been derived.

In [1], considering the Schur-convexity or Schur-concavity of the functions $s_{\alpha}^{*}(G)$ and using additional information on the localization of the eigenvalues, the following Theorems, which generalize Theorem 3.3 in [7], have been proved.

Theorem 2.1. Let $G$ be a simple connected graph with $n \geq 3$ vertices and $\lambda_{1} \geq \theta$ :

1. if $\alpha<0$ or $\alpha>1$ then

$$
s_{\alpha}^{*}(G) \geq \theta^{\alpha}+\frac{(n-\theta)^{\alpha}}{(n-2)^{\alpha-1}}
$$

2. if $0<\alpha<1$ then

$$
s_{\alpha}^{*}(G) \leq \theta^{\alpha}+\frac{(n-\theta)^{\alpha}}{(n-2)^{\alpha-1}} .
$$

Theorem 2.2. Let $G$ be a simple connected graph with $n \geq 4$ vertices which is not complete and $\lambda_{1} \geq \theta, \lambda_{2} \geq \beta$ with $\theta \geq \beta$ and $\theta+\beta(n-2)>n$.

1. if $\alpha<0$ or $\alpha>1$ then

$$
s_{\alpha}^{*}(G) \geq \theta^{\alpha}+\beta^{\alpha}+\frac{(n-\theta-\beta)^{\alpha}}{(n-3)^{\alpha-1}}
$$

2. if $0<\alpha<1$ then

$$
s_{\alpha}^{*}(G) \leq \theta^{\alpha}+\beta^{\alpha}+\frac{(n-\theta-\beta)^{\alpha}}{(n-3)^{\alpha-1}} .
$$

It is noteworthy to state that the results in Theorem 2.2 are tighter than those in Theorem 2.1 (for more details see [3] and [4]).

In [7], the bounds in Theorem 2.1 have been previously proved identifying $\theta$ as

$$
P=1+\sqrt{\frac{2}{n(n-1)} \sum_{(i, j \in E)} \frac{1}{d_{i} d_{j}}} .
$$

In Section 4, by applying some results proved in [5], we provide lower bounds of $\lambda_{1}$ and $\lambda_{2}$ that enable us to assess tighter bounds of $s_{\alpha}^{*}(G)$ than in [7]. In particular, we obtain an alternative value of $\theta$ than (2.5) (referred as $Q$ ) and a specific value of $\beta$ (referred as $R$ ). 


\section{A nonlinear optimization problem to bound eigenvalues}

We now recall a methodology based on majorization techniques (see [5] and [15]) that allows us to find a suitable localization of $\lambda_{1}$ and $\lambda_{2}$.

At this regard, we define the set

$$
S_{b}^{\lambda}=\left\{\boldsymbol{\lambda} \in \mathbb{R}_{+}^{n-1}: \lambda_{1} \geq \lambda_{2} \geq \ldots \geq \lambda_{n-1} \geq 0, \sum_{i=1}^{n-1} \lambda_{i}=n, g(\boldsymbol{\lambda})=\sum_{i=1}^{n-1} \lambda_{i}^{p}=b\right\},
$$

where $p$ is an integer greater than $1, b \in \mathbb{R}$ and $g$ is a continuous function, homogeneous of degree $p$, real and strictly Schur-Convex (see [15]).

The following fundamental lemma holds (see Lemma 2.1 in [5]):

Lemma 3.1. Fix $b \in \mathbb{R}$ and consider the set $S_{b}^{\lambda}$. Then either $b=\frac{n^{p}}{(n-1)^{p-1}}$ or there exists a unique integer $1 \leq h^{*}<(n-1)$ such that:

$$
\frac{n^{p}}{\left(h^{*}+1\right)^{p-1}}<b \leq \frac{n^{p}}{\left(h^{*}\right)^{p-1}},
$$

where $h^{*}=\left\lfloor\sqrt[p-1]{\frac{n^{p}}{b}}\right\rfloor$

A lower bound for $\lambda_{h}(h=1, \ldots, n-1)$ can be obtained by solving the following optimization problem $P^{*}(h)$ :

$$
\min \left(\lambda_{h}\right) \text { subject to } \lambda \in S_{b}^{\lambda}
$$

The solution of the nonlinear optimization problem $P^{*}(h)$ is given in the following Theorem that we recall from Theorem 3.2 in [5] (for detailed proof see [5]).

Theorem 3.2. The solution of the optimization problem $P^{*}(h)$ is $\left(\frac{n}{n-1}\right)$ if $b=\frac{n^{p}}{(n-1)^{p-1}}$. If $b \neq \frac{n^{p}}{(n-1)^{p-1}}$, the solution of the optimization problem $P^{*}(h)$ is $\delta^{*}$ where

1. for $h=1, \delta^{*}$ is the unique root of the equation

$$
f(\delta, p)=h^{*} \delta^{p}+\left(n-h^{*} \delta\right)^{p}-b=0
$$

in $I=\left(\frac{n}{h^{*}+1}, \frac{n}{h^{*}}\right]$;

2. for $1<h \leq\left(h^{*}+1\right)$, $\delta^{*}$ is the unique root of the equation

$$
f(\delta, p)=(n-h) \delta^{p}+(h-1) \frac{(n-(n-h) \delta)^{p}}{(h-1)^{p}}-b=0
$$

in $I=\left(0, \frac{n}{n-1}\right]$;

3. for $h>\left(h^{*}+1\right), \delta^{*}$ is zero.

It is noteworthy that we use this Theorem in the next Section to obtain lower bounds for $\lambda_{1}$ and $\lambda_{2}$. 


\section{New bounds for normalized Laplacian eigenvalues and $s_{\alpha}^{*}(G)$}

We now present a schematic framework of the main steps we follow in order to provide new limitations for $\lambda_{1}$ and $\lambda_{2}$ useful to get new bounds for the descriptor $s_{\alpha}^{*}(G)$.

\section{A new lower bound $Q$ for $\lambda_{1}$}

At this regard, we consider Theorem 3.2 limiting $^{1}$ the analysis when $p=2$ : in this case we know indeed that $b=n+2 \sum_{(i, j) \in E} \frac{1}{d_{i} d j}$. For Lemma 3.1, when $b=\frac{n^{2}}{(n-1)}$ the solution of optimization problem $P^{*}(h)$ is $\left(\frac{n}{n-1}\right)$. This is the case of the complete graph $K_{n}$. Instead, when $b \neq \frac{n^{2}}{(n-1)}, h^{*}=\left\lfloor\frac{n^{2}}{b}\right\rfloor$.

Considering non-complete graphs, to get a lower bound for $\lambda_{1}$ we solve Equation (3.2) being $h=1$. By some basic algebra, the acceptable solution in the interval $I$ is equal to $\delta^{*}=\frac{\left(n+\sqrt{\frac{b\left(h^{*}+1\right)-n^{2}}{h^{*}}}\right)}{\left(1+h^{*}\right)}$ and we refer to this value as $Q$.

2. New bounds for $s_{\alpha}^{*}(G)$ based on $Q$

Considering Theorem 2.1 we obtain new bounds for $s_{\alpha}^{*}(G)$ by replacing the generic limitation $\theta$ with $Q$ in (2.1) and (2.2).

\section{Comparison between $Q$ and $P$}

The value of $Q$ can be compared to $P$ (see Equation (2.5)) in order to show how bounds (2.1) and (2.2), computed by assuming $\theta=Q$, perform better than those with $\theta=P$ (as proposed in [7]). It is well known that, for every connected graph of order $n$ (see [2]), we have:

$$
\frac{1}{n-1} \leq \frac{2}{n} \sum_{(i, j) \in E} \frac{1}{d_{i} d_{j}}<1
$$

and the left inequality is attained for the complete graph $G=K_{n}$.

Figure 1 reports patterns of $P$ and $Q$, varying the quantity $t=2 \cdot \sum_{(i, j) \in E} \frac{1}{d_{i} d_{j}}$ in the proper interval $\left(\frac{n}{n-1}, n\right)$ (see Equation (4.1)) for alternative values of number of vertices $n$. Being $P=1+\sqrt{\frac{t}{n(n-1)}}$, it is easy to see that $P$ has a monotonic behaviour with respect to $t . P \in\left(\frac{n}{n-1}, 1+\sqrt{\frac{1}{n-1}}\right)$, while $Q \in\left(\frac{n}{n-1}, 2\right)$. Furthermore, Figure 1 shows that $Q$ increases faster than $P$ when $t \in\left(\frac{n}{n-1}, n\right)$.

\footnotetext{
${ }^{1}$ For values of $p \neq 2, b$ depends on the graph's structure and topology. So the procedure can be only numerically applied: we need to compute the eigenvalues of normalized Laplacian matrix, but this information allows to directly obtain $s_{\alpha}^{*}(G)$. In this case, the evaluation of bounds is useless.
} 

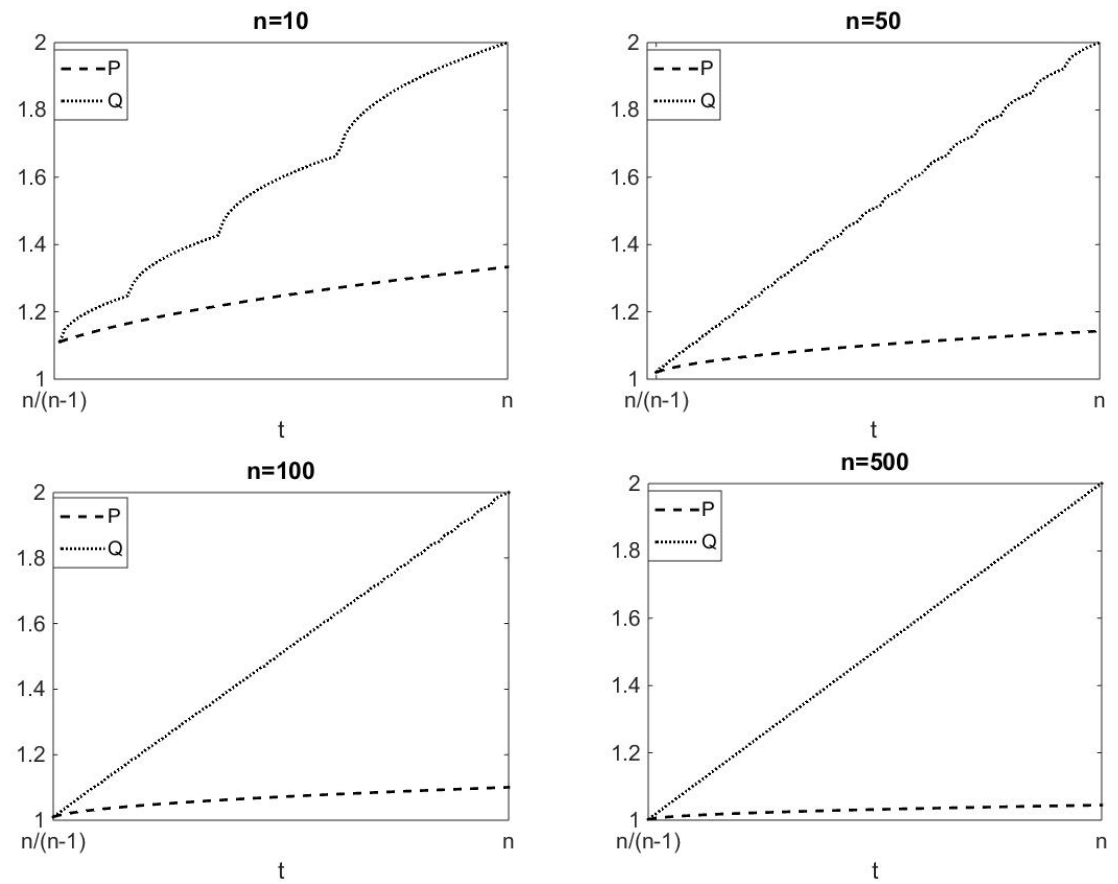

Figure 1: $Q$ and $P$ according to different values of $t \in\left(\frac{n}{n-1}, n\right)$ and several number of vertices.

Going deeply into the analysis, we now analytically prove that our bound $Q$ on $\lambda_{1}$ is always better than bound $P$ provided in [7]

Main Result. The limitation $Q$ is strictly greater than $P$ for non-complete graphs.

Proof. We start considering:

$$
f(t)=Q-P=\frac{\left(n+\sqrt{\frac{(n+t)\left(h^{*}+1\right)-n^{2}}{h^{*}}}\right)}{\left(1+h^{*}\right)}-1-\sqrt{\frac{t}{n(n-1)}},
$$

where $f(t) \in\left(0,1-\sqrt{\frac{t}{n(n-1)}}\right), h^{*}=\left\lfloor\frac{n^{2}}{n+t}\right\rfloor$ and $h^{*} \in\left(\left\lfloor\frac{n}{2}\right\rfloor, n-1\right)$.

Furthermore, when

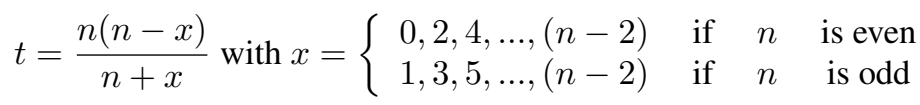

$\frac{n^{2}}{n+t}$ is an integer (i.e. $\left.h^{*}=\frac{n^{2}}{n+t}\right)$.

We can now distinguish two cases:

- when (4.3) holds, $\frac{n^{2}}{n+t}$ is an integer. Doing some algebra, we have $f(t)=$ $\frac{t}{n}-\sqrt{\frac{t}{n(n-1)}}$. It is immediate to see that $f(t)>0$, with $f(t)=0$ only for complete graphs (being in that case $t=\frac{n}{n-1}$ ); 
- otherwise, $\frac{n^{2}}{n+t}$ is not an integer. Being $f(t) \geq 0$ at the boundaries of its domain and $f(t)>0$ for values of $t$ so that $h^{*}$ is an integer, by proving that $f(t)$ is strictly increasing on its remaining domain, we have $f(t)>0$. Since $f(t)$ is required to be strictly increasing, we compute $f^{\prime}(t)=\frac{1}{2 h^{*} \sqrt{\frac{(n+t)\left(h^{*}+1\right)-n^{2}}{h^{*}}}}-\frac{1}{2 n(n-1)} \frac{1}{\sqrt{\frac{t}{n(n-1)}}}$ (defined in $\left.t \neq \frac{n(n-x)}{n+x}\right)$.

In order to show that $f^{\prime}(t)>0$, by simple algebra we get: $t\left(n(n-1)-h^{*}\left(h^{*}+1\right)\right)+n h^{*}\left(n-\left(h^{*}+1\right)\right)>0$.

Being $h^{*}<n-1$ for non-complete graphs, we have $f^{\prime}(t)>0$.

It follows that $Q>P$ for non-complete graphs.

Now, $\lambda_{1} \geq Q \geq P$ entails that bounds (2.1) and (2.2) with $\theta=Q$ perform better than those in [7] (see [3] and [4] for more theoretical details).

\section{A new lower bound $\boldsymbol{R}$ for $\boldsymbol{\lambda}_{2}$}

With the aim to improve previous results, we can now derive additional information on $\lambda_{2}$. We still apply Theorem 3.2, considering the case $h=2$. Since $h \leq\left(h^{*}+1\right)$, we solve the Equation (3.3) finding in the interval $I$ the acceptable solution:

$$
R=\delta^{*}=\frac{n-\sqrt{\frac{b(n-1)-n^{2}}{n-2}}}{n-1} .
$$

\section{New bounds for $s_{\alpha}^{*}(G)$ based on $Q$ and $R$}

Considering Theorem 2.2, it is possible to obtain new bounds for $s_{\alpha}^{*}(G)$ by replacing the generic limitations $\theta$ and $\beta$ with $Q$ and $R$ respectively in (2.3) and (2.4). In order to assess these bounds, both conditions of Theorem 2.2 must be satisfied.

In this case, the leftmost inequality of (4.1) implies $b \geq \frac{n^{2}}{n-1}$. By plugging this information in the value of $R$, we easily obtain $R \leq \frac{n}{n-1}$ that fulfills the condition $R \leq Q$ of Theorem 2.2. The other condition, $Q+R(n-2)>n$, required by Theorem 2.2 will be numerically checked in the next Section.

In case of bipartite graphs it is well known that $\lambda_{1}=2$. If we set $\theta=2$ in Theorem 2.1, we derive the same results found in [1]. Furthermore, by placing $\theta=2$ and $\beta=R$ in bounds (2.3) and (2.4), we also provide limitations for bipartite graphs.

\section{Some numerical results}

The proposed bounds have been evaluated on different graphs. We now focus only on nonbipartite graphs and we provide a comparison with literature (see [7]).

In order to assure a robust analysis, graphs have been randomly generated following the Erdös-Rényi (ER) model $G_{E R}(n, q)$ (see [6], [8], [11] and [12]). Graphs have been obtained by using a MatLab code that gives back only connected graph based on the ER model (see [9] and [10]). In this fashion, the graph is constructed by connecting nodes randomly such that edges are included with probability independent from every other edge. The results are based on a classic assumption of a probability of existence of edges $q$ equal to 0.5 . We obtain indeed that the generated graphs have a number of edges not far from the half of its maximum value as proved in the literature (see for example [13]). 
At this regard, in Table $1, s_{\alpha}^{*}(G)$ has been computed for several graphs by fixing $\alpha$ equal to 0.5 . We report values of upper bound (2.2) evaluated by using $\theta=Q$ or $\theta=P$ (as proposed in [7]).

We refer to these bounds as (2.2Q) and (2.2P). Likewise bound (2.4QR) identifies bound (2.4) evaluated when $\theta=Q$ and $\beta=R$, where the results has been provided assuring that assumptions of Theorem 2.2 are satisfied. Relative errors $r$ measures the absolute value of the difference between the upper bounds and $s_{\alpha}^{*}(G)$ divided by the value of $s_{\alpha}^{*}(G)$. We observe an improvement with respect to existing bounds according to all the analyzed graphs and the improvement appears reduced for very large graphs. However, for large graphs the formula provided in [7] already gives a very low relative error.

\begin{tabular}{|c|c|c||c|c|c|c|c|c|c||}
\hline \hline$n$ & $d_{1}$ & $m$ & $s_{\alpha}^{*}(G)$ & bound $(2.2 Q)$ & bound $(2.4 Q R)$ & bound $(2.2 P)$ & $\mathrm{r}(2.2 Q)$ & $\mathrm{r}(2.4 Q R)$ & $\mathrm{r}(2.2 P)$ \\
\hline \hline 4 & 2 & 3 & 3.35 & 3.44 & 3.43 & 3.46 & $2.86 \%$ & $2.55 \%$ & $3.47 \%$ \\
\hline 5 & 4 & 9 & 4.46 & 4.47 & 4.47 & 4.47 & $0.23 \%$ & $0.21 \%$ & $0.25 \%$ \\
\hline 6 & 3 & 6 & 5.30 & 5.47 & 5.46 & 5.48 & $3.13 \%$ & $3.00 \%$ & $3.27 \%$ \\
\hline 7 & 5 & 14 & 6.43 & 6.48 & 6.48 & 6.48 & $0.83 \%$ & $0.81 \%$ & $0.86 \%$ \\
\hline 8 & 5 & 13 & 7.33 & 7.48 & 7.48 & 7.48 & $2.02 \%$ & $1.98 \%$ & $2.06 \%$ \\
\hline 9 & 6 & 16 & 8.31 & 8.48 & 8.48 & 8.48 & $2.04 \%$ & $2.01 \%$ & $2.07 \%$ \\
\hline 10 & 8 & 25 & 9.39 & 9.51 & 9.48 & 9.52 & $1.36 \%$ & $1.04 \%$ & $1.37 \%$ \\
\hline 20 & 15 & 95 & 19.37 & 19.51 & 19.49 & 19.51 & $0.71 \%$ & $0.62 \%$ & $0.72 \%$ \\
\hline 30 & 19 & 209 & 29.36 & 29.50 & 29.50 & 29.50 & $0.49 \%$ & $0.46 \%$ & $0.49 \%$ \\
\hline 50 & 33 & 604 & 49.37 & 49.50 & 49.50 & 49.50 & $0.27 \%$ & $0.26 \%$ & $0.27 \%$ \\
\hline 100 & 60 & 2459 & 99.37 & 99.50 & 99.50 & 99.50 & $0.13 \%$ & $0.12 \%$ & $0.13 \%$ \\
\hline 200 & 116 & 10001 & 199.38 & 199.50 & 199.50 & 199.50 & $0.06 \%$ & $0.05 \%$ & $0.06 \%$ \\
\hline 300 & 179 & 22437 & 299.37 & 299.50 & 299.50 & 299.50 & $0.04 \%$ & $0.04 \%$ & $0.04 \%$ \\
\hline 500 & 279 & 62456 & 499.38 & 499.50 & 499.50 & 499.50 & $0.03 \%$ & $0.02 \%$ & $0.03 \%$ \\
\hline \hline
\end{tabular}

Table 1: Upper bounds for $s_{\alpha}^{*}(G)$ for $\alpha=0.5$ and relative errors.

The comparison has been extended in order to test the behaviour of the upper bounds on alternative graphs. First of all, in the ER model used to generate graphs, the parameter $q$ can be thought of as a weighting function. As $q$ increases from 0 to 1 , the model becomes more and more likely to include graphs with more edges and less and less likely to include graphs with fewer edges. In this regard, we assign several values of $q$ moving from the default value of 0.5 . For sake of simplicity we report only the relative errors derived for graphs generated by using respectively $q=0.1$ and $q=0.9$ (see Figure 2). In all cases bound $(2.4 Q R)$ assures the best approximation to $s_{\alpha}^{*}(G)$ for $\alpha=0.5$. We observe a best behaviour of all bounds when $q=0.9$ because we are moving towards the complete graph. We have indeed that the density of the graphs increases as long as greater probabilities are considered.
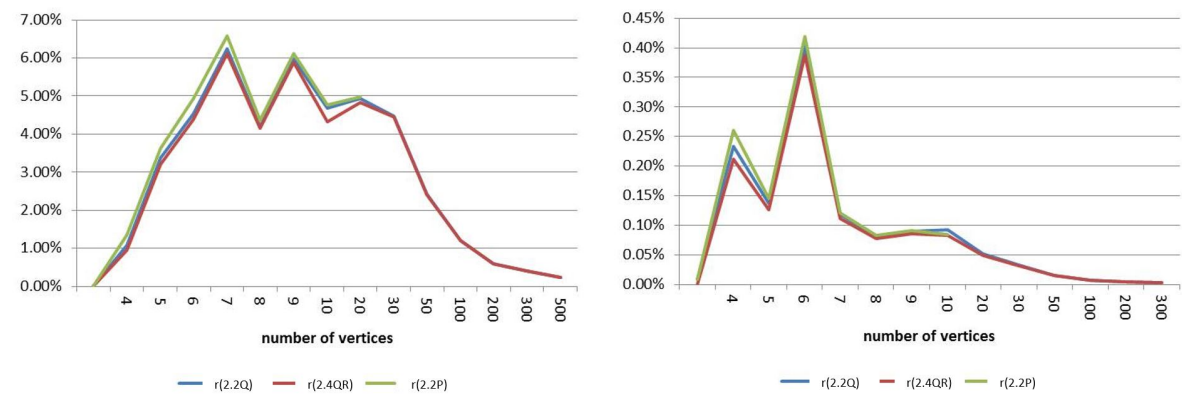

Figure 2: Relative errors of upper bounds of $s_{0.5}^{*}(G)$ for graphs $E R(n, 0.1)$ and $E R(n, 0.9)$ respectively. 
Finally, for the same index $s_{0.5}^{*}(G)$, upper bounds have been evaluated for trees ${ }^{2}$. Table 2 depicts slighter differences for larger graphs in this case too. However it could be noticed how the relative improvement of bounds respect to other bounds is greater than in case of non-bipartite graphs. Despite greater relative errors are observed, bound $(2.4 Q R)$ is confirmed as the tighter bound also in this case.

\begin{tabular}{|c||c|c|c|c|}
\hline \multicolumn{1}{|c||}{} & \multicolumn{4}{c|}{ Trees } \\
\hline$n$ & $s_{\alpha}^{*}(G)$ & $\mathrm{r}(2.2 Q)$ & $\mathrm{r}(2.4 Q R)$ & $\mathrm{r}(2.2 P)$ \\
\hline \hline 4 & 3.35 & $2.04 \%$ & $1.95 \%$ & $3.47 \%$ \\
\hline 5 & 4.32 & $2.17 \%$ & $2.14 \%$ & $3.48 \%$ \\
\hline 6 & 5.23 & $3.56 \%$ & $3.51 \%$ & $4.73 \%$ \\
\hline 7 & 6.19 & $3.62 \%$ & $3.59 \%$ & $4.67 \%$ \\
\hline 8 & 7.15 & $3.67 \%$ & $3.64 \%$ & $4.61 \%$ \\
\hline 9 & 8.22 & $2.35 \%$ & $2.34 \%$ & $3.20 \%$ \\
\hline 10 & 8.85 & $6.35 \%$ & $6.32 \%$ & $7.15 \%$ \\
\hline 20 & 18.07 & $7.45 \%$ & $7.44 \%$ & $7.88 \%$ \\
\hline 30 & 27.63 & $6.47 \%$ & $6.47 \%$ & $6.77 \%$ \\
\hline 50 & 45.73 & $8.07 \%$ & $8.06 \%$ & $8.25 \%$ \\
\hline 100 & 91.23 & $8.97 \%$ & $8.97 \%$ & $9.06 \%$ \\
\hline 200 & 182.72 & $9.14 \%$ & $9.14 \%$ & $9.18 \%$ \\
\hline 300 & 274.71 & $8.99 \%$ & $8.99 \%$ & $9.02 \%$ \\
\hline 500 & 457.71 & $9.11 \%$ & $9.11 \%$ & $9.13 \%$ \\
\hline
\end{tabular}

Table 2: $s_{0.5}^{*}(G)$ and relative errors for Trees $T$.

The analysis has been further developed considering a value of $\alpha$ equal to 1.5. Generating a similar sample of graphs, both $s_{1.5}^{*}(G)$ and the relative bounds have been derived. For sake of simplicity we report only the results for $E R(n, 0.5)$ observing that the additional information on the localization of $\lambda_{1}$ and $\lambda_{2}$ lead to the tighter lower bound (2.3QR). Analogous results have been obtained by considering both ER graphs with alternative values of $q$ and bipartite graphs.

\begin{tabular}{|c|c|c||c|c|c|c||c|c|c||}
\hline \hline$n$ & $d_{1}$ & $m$ & $s_{\alpha}^{*}(G)$ & bound $(2.1 Q)$ & bound $(2.3 Q R)$ & bound $(2.1 P)$ & $\mathrm{r}(2.1 Q)$ & $\mathrm{r}(2.3 Q R)$ & $\mathrm{r}(2.1 P)$ \\
\hline \hline 4 & 3 & 4 & 4.79 & 4.66 & 4.67 & 4.62 & $2.69 \%$ & $2.39 \%$ & $3.49 \%$ \\
\hline 5 & 2 & 4 & 6.22 & 5.65 & 5.69 & 5.60 & $9.07 \%$ & $8.51 \%$ & $9.95 \%$ \\
\hline 6 & 4 & 9 & 6.85 & 6.59 & 6.60 & 6.57 & $3.78 \%$ & $3.63 \%$ & $4.00 \%$ \\
\hline 7 & 6 & 13 & 7.77 & 7.57 & 7.57 & 7.56 & $2.56 \%$ & $2.49 \%$ & $2.65 \%$ \\
\hline 8 & 7 & 18 & 8.75 & 8.56 & 8.56 & 8.55 & $2.15 \%$ & $2.10 \%$ & $2.20 \%$ \\
\hline 9 & 4 & 12 & 10.28 & 9.58 & 9.59 & 9.55 & $6.88 \%$ & $6.79 \%$ & $7.15 \%$ \\
\hline 10 & 7 & 26 & 10.83 & 10.52 & 10.55 & 10.51 & $2.90 \%$ & $2.60 \%$ & $2.94 \%$ \\
\hline 20 & 13 & 98 & 20.88 & 20.50 & 20.52 & 20.50 & $1.81 \%$ & $1.71 \%$ & $1.81 \%$ \\
\hline 30 & 19 & 222 & 30.88 & 30.50 & 30.51 & 30.50 & $1.21 \%$ & $1.18 \%$ & $1.21 \%$ \\
\hline 50 & 31 & 644 & 50.85 & 50.50 & 50.51 & 50.50 & $0.68 \%$ & $0.67 \%$ & $0.68 \%$ \\
\hline 100 & 62 & 2512 & 100.87 & 100.50 & 100.50 & 100.50 & $0.37 \%$ & $0.36 \%$ & $0.37 \%$ \\
\hline 200 & 117 & 9918 & 200.88 & 200.50 & 200.50 & 200.50 & $0.19 \%$ & $0.19 \%$ & $0.19 \%$ \\
\hline 300 & 179 & 22540 & 300.87 & 300.50 & 300.50 & 300.50 & $0.12 \%$ & $0.12 \%$ & $0.12 \%$ \\
\hline 500 & 279 & 62063 & 500.88 & 500.50 & 500.50 & 500.50 & $0.08 \%$ & $0.08 \%$ & $0.08 \%$ \\
\hline
\end{tabular}

Table 3: Lower bounds for $s_{1.5}^{*}(G)$ and absolute value of relative errors.

\section{Conclusions}

In this paper we provide tighter bounds for the sum of the $\alpha$-power of the non-zero normalized Laplacian eigenvalues taking into account additional information on the localization of the eigenvalues of the normalized Laplacian matrix of the graph, $\mathcal{L}(G)$. To this aim lower bounds of the eigenvalues are derived by means of the solution of a class of suitable nonlinear optimization problems based on majorization techniques. We provide indeed closed

\footnotetext{
${ }^{2}$ Tree has been generated by using Prüfer code. The Prüfer sequence of a labeled tree is a unique sequence associated to the tree. The sequence for a tree on $n$ vertices has length $n-2$ and it can be generated by a simple iterative algorithm. It is a way to map bijectively trees on $n$ vertices into $n-2$ long sequences of integers drawn from $n$.
} 
formulae that allow to compute upper and lower bounds of $s_{\alpha}^{*}(G)$ by using the additional information on the first and the second eigenvalue of $\mathcal{L}(G)$. It is noteworthy that even only considering the limitation on the first normalized Laplacian eigenvalue we improve existing bounds of $s_{\alpha}^{*}(G)$ for non-complete graphs. Numerical comparisons confirm how bounds based also on the second normalized Laplacian eigenvalue are former than those presented in literature. In particular, the analysis has been developed randomly generating both bipartite and non-bipartite graphs with a different number of vertices.

\section{References}

[1] M. Bianchi, A. Cornaro, J. Palacios and A. Torriero, Bounding the Sum of Powers of Normalized Laplacian Eigenvalues of Graphs through Majorization Methods, MATCH Commun. Math. Comput. Chem. 70(2) (2013), 707-716.

[2] M. Bianchi, A. Cornaro, J. Palacios and A. Torriero, Bounds for the Kirchhoff index via majorization techniques, J. Math. Chem. 51(2) (2013), 569-587.

[3] M. Bianchi, A. Cornaro and A. Torriero, A majorization method for localizing graph topological indices, Discrete Appl. Math. 161 (2013), 2731-2739.

[4] M. Bianchi, A. Cornaro and A. Torriero, Majorization under constraints and bounds of the second Zagreb index, Mathematical Inequalities and Applications 16(2) (2013), 329-347.

[5] M. Bianchi and A. Torriero, Some localization theorems using a majorization technique, Journal of Inequalities and Applications 5 (2000), 443-446.

[6] B. Bollobás, Random Graphs, Cambridge Univ. Press, London, 2001.

[7] S. Bozkurt and D. Bozkurt, On the sum of powers of normalized laplacian eigenvalues of graphs, MATCH Commun. Math. Comput. Chem. 68 (2012), 917-930.

[8] F. R. K. Chung, L. Lu and V. Vu., The spectra of random graphs with given expected degrees, Proceedings of the National Academy of Sciences (2003), 6313-6318.

[9] G. P. Clemente and A. Cornaro, Computing Lower Bounds for the Kirchhoff Index Via Majorization Techniques, MATCH Commun. Math. Comput. Chem. 73 (2015), 175-193.

[10] A. Cornaro and G. P. Clemente, A New Lower Bound for the Kirchhoff Index using a numerical procedure based on Majorization Techniques, Electronic Notes in Discrete Mathematics 41 (2013), 383-390.

[11] P. Erdős and A. Rényi, On Random Graphs I, Publicationes Mathematicae 6 (1959), 290-297.

[12] P. Erdős and A. Rényi, On the evolution of random graphs, Publications of the Mathematical Institute of the Hungarian Academy of Sciences 5 (1960), 17-61.

[13] E. Estrada, The Structure of Complex Networks: Theory and Applications, Oxford Univ. Press, New York, 2011.

[14] M. Liu and B. Liu, A note on sum of powers of the Laplacian eigenvalues of a graphs, Appl. Math. Lett. 24 (2011), 249-252.

[15] A. W. Marshall, I. Olkin and B. Arnold, Inequalities: Theory of Majorization and Its Applications, Springer, 2011.

[16] G. X. Tian, T. Z. Huang and B. Zhou, A note on sum of powers of the Laplacian eigenvalues of bipartite graphs, Linear Algebra and its Applications 430 (2009), 2503-2510.

[17] R. J. Wilson, Introduction to graph theory, Addison Wesley, 1996.

[18] B. Zhou, On a sum of powers of the Laplacian eigenvalues of a graphs, Linear Algebra and its Applications 429 (2008), 2239-2246. 
G. P. Clemente and A. Cornaro: New bounds for the sum of powers of normalized Laplacian... 413

[19] B. Zhou, On a sum of powers of Laplacian eigenvalues and Laplacian Estrada index of graphs, MATCH Common. Math. Comput. Chem. 62 (2009), 611-619.

[20] B. Zhou and A. Ilić, On a sum of powers of Laplacian eigenvalues of bipartite graphs, Czech. Math. J. 60 (2010), 1161-1169. 